\title{
The History of the Equatorial Emission in SS433
}

\author{
Ralph Spencer ${ }^{1}$ \\ Jodrell Bank Observatory \\ The University of Manchester, Macclesfield, SK11 9DL, UK \\ E-mail:res@jb.man.ac.uk
}

\begin{abstract}
The equatorial radio emission recently discovered in SS433 adds a further intriguing feature to what is already a bizarre object. Radio images of SS433 taken over the last 25 years were examined to find that evidence for the existence of the emission exists even in the early images. The extended emission is found in 18 out of 78 separate observations, with a higher detection rate in recent years, reflecting improving imaging capability of interferometer arrays. The position angle of the emission changes, being on average perpendicular to the precession axis of the jets and varying over a similar range to that of the jets, however there is not a close relationship between the jet and equatorial emission position angle. This suggests that if the emission is formed by a precessing equatorial wind then the wind velocity must vary. Alternatively the emission could be due to colliding back flow from the jets.
\end{abstract}

VI Microquasar Workshop: Microquasars and Beyond Societa' del Casino, Como, Italy 18-22 September, 2006

\footnotetext{
$1 \quad$ Speaker
} 


\section{Introduction}

SS433 is famous for its moving optical lines [13] and precessing radio jets [23,10]. Recently $[16,17,1,21]$ radio emission was found in a direction roughly perpendicular to the precession axis (position angle $100^{\circ}[10,23,24]$ ) of the radio jets, in diffuse 'ruff' structure. The observations of Blundell et al. (2001) [1] suggest that the emission is thermal in origin, though with a high brightness temperature, though Paragi et al. 2001 [18] find that the emission in compact regions may be non-thermal. The existence of an excretion disk surrounding the system has been suggested [3], and the disk may manifest itself by this equatorial emission. If so we would expect the emission to be persistent, and be present in earlier images made of the jets in SS433. High resolution radio imaging fidelity has improved greatly over recent years, with the advent of phase referencing, better calibration and imaging algorithms and improved uv-plane coverage with instruments like the VLBA and EVN. The early images were originally interpreted conservatively, and so features might have been missed. All the published images of SS433 were therefore examined with the benefit of hindsight.

\section{The Archive}

A total of 78 epochs of radio maps were found in the literature from 1979 to 2003. Most of the observations were made using VLBI, MERLIN or the VLA as stand alone instruments, though there are several cases where observations were made with multiple arrays. The beautiful VLBA movie of Mioduszewski et al (http://www.aoc.nrao.edu/ amiodusz/) [21] has been counted as one epoch here, though really there were 39 separate images, however the equatorial emission was present in nearly all the images. The discovery paper for the radio jets only used a single baseline interferometer and so earlier (1976) images are not available [23]. The 78 images used are listed in table 1, together with the observing frequency, the instrument and the reference. The 18 cases where there is evidence of equatorial emission indicated by an asterisk in column 1 . The evidence varies from a slight extension in the contours in a general $\mathrm{N}-\mathrm{S}$ direction near the centre of the object, to clearer cases where there is discrete emission. An example of the former can be seen in figure 1, where is there is emission to the South of the core. A case where there is clear emission on the North and South of the core region is shown in figure 2, using combined MERLIN and EVN data. It is clear that the equatorial emission was indeed present in the images, though it is not surprising it was missed given the difficulties of mapping low declination sources where the uv coverage of each baseline is dominantly E-W. The fraction of epochs were the emission is present is clearly higher in recent years, reflecting improvements in the imaging capability of radio interferometers. 
Table 1 Radio Observations of SS433

\begin{tabular}{|c|c|c|c|}
\hline $\begin{array}{c}\text { Date of } \\
\text { Observations }\end{array}$ & $\begin{array}{c}\text { Frequency } \\
\text { GHz }\end{array}$ & Instrument & Reference \\
\hline 22/07/1979 & 1.5 & VLA & Gilmore \& Seaquist 1980 [6] \\
\hline 22/07/1979 & 4.9 & VLA & Gilmore \& Seaquist 1980 [6] \\
\hline 08/09/1979 & 4.9 & VLA & Hjellming \& Johnston 1981 [10] \\
\hline 16/09/1979 & 4.9 & VLA & Hjellming \& Johnston 1981 [10] \\
\hline 07/12/1979 & 4.9 & VLA & Hjellming \& Johnston 1981,1981b [10,11] \\
\hline 09/01/1980 & 5 & EVN & Schilizzi et al. 1982 [22] \\
\hline 02/02/1980 & 0.4 & MERLIN & Spencer 1984 [24] \\
\hline 20/02/1980 & 4.9 & VLA & Gilmore et al $1981[7]$ \\
\hline 07/03/1980 & 4.9 & VLA & Hjellming \& Johnston 1981,1981b [10,11] \\
\hline 05/04/1980* & 4.9 & VLA & Hjellming \& Johnston 1981,1981b [10,11] \\
\hline $19 / 05 / 1980 *$ & 4.9 & VLA & Gilmore et al. 1981 [7] \\
\hline 20/06/1980 & 4.9 & VLA & Hjellming \& Johnston 1981,1981b [10,11] \\
\hline $14 / 08 / 1980$ & 1.7 & MERLIN & Spencer $1984[7]$ \\
\hline 02/09/1980 & 1.7 & MERLIN & Spencer 1984 [7] \\
\hline 03/10/1980 & 5 & EVN & Romney et al. 1987 [20] \\
\hline 05/10/1980 & 2.3 & VLBI & Niell et al. 1982 [15] \\
\hline $12 / 10 / 1980$ & 1.7 & MERLIN & Spencer 1984 [24] \\
\hline 25/10/1980 & 1.7 & MERLIN & Spencer 1984 [24] \\
\hline $16 / 12 / 1980$ & 1.4 & EVN & Romney et al 1987 [20] \\
\hline $13 / 02 / 1981$ & 2.3 & VLBI & Niell et al. 1982 [15] \\
\hline $15 / 02 / 1981$ & 1.7 & EVN & Romney et al. 1987 [20] \\
\hline $11 / 04 / 1981^{*}$ & 5 & EVN & Romney et al. 1987 [20] \\
\hline 17/05/1981 & 2.3 & VLBI & Niell et al. 1982 [15] \\
\hline 26/05/1981 & 5 & EVN & Romney et al. 1987 [20] \\
\hline 01/06/1981 & 5 & EVN & Romney et al. 1987 [20] \\
\hline 30/08/1981* & 1.4 & EVN & Romney et al. 1987 [20] \\
\hline 09/12/1981 & 5 & EVN & Romney et al. 1987 [20] \\
\hline 04/04/1982 & 5 & MERLIN & Spencer \& Waggett 1984 [25] \\
\hline 12/04/1982 & 5 & MERLIN & Spencer \& Waggett 1984 [25] \\
\hline 20/04/1982 & 5 & MERLIN & Spencer \& Waggett 1984 [25] \\
\hline 28/04/1982 & 5 & MERLIN & Spencer \& Waggett 1984 [25] \\
\hline 14/05/1982 & 5 & MERLIN & Spencer \& Waggett 1984 [25] \\
\hline 25/05/1982 & 15 & VLA & Heavens et al. 1990 [9] \\
\hline 27/05/1982 & 5 & MERLIN & Spencer \& Waggett 1984 [25] \\
\hline 13/06/1982 & 5 & MERLIN & Spencer \& Waggett 1984 [25] \\
\hline 02/07/1982 & 5 & MERLIN & Spencer \& Waggett 1984 [25] \\
\hline $13 / 06 / 1984$ & 1.4 & EVN & Fejes $1986[4]$ \\
\hline 17/05/1985 & 5 & $\mathrm{EVN}$ & Vermeulen et al. 1987 [30] \\
\hline 19/05/1985 & 5 & EVN & Vermeulen et al. 1987 [30] \\
\hline 21/05/1985 & 5 & EVN & Vermeulen et al. 1987 [30] \\
\hline 23/05/1985 & 5 & EVN & Vermeulen et al. 1987 [30] \\
\hline 25/05/1985 & 5 & EVN & Vermeulen et al. 1987 [30] \\
\hline 27/05/1985 & 5 & EVN & Vermeulen et al. 1987 [30] \\
\hline 15/03/1986 & 1.7 & EVN & Fejes et al. 1988 [5] \\
\hline 23/05/1987 & 5 & EVN & Vermeulen et al. 1993 [31] \\
\hline 25/05/1987 & 5 & EVN & Vermeulen et al 1993 [31] \\
\hline
\end{tabular}




\begin{tabular}{|c|c|c|c|}
\hline 27/05/1987 & 5 & EVN & Vermeulen et al 1993 [31] \\
\hline 29/05/1987 & 5 & EVN & Vermeulen et al 1993 [31] \\
\hline 31/05/1987 & 5 & $\mathrm{EVN}$ & Vermeulen et al 1993 [31] \\
\hline 02/06/1987 & 5 & $\mathrm{EVN}$ & Vermeulen et al 1983 [31] \\
\hline 17/07/1987* & 5,15 & VLA & Stirling et al. 2004 [29] \\
\hline 30/04/1988 & 1.7 & MERLIN & Spencer et al. 1993 [26] \\
\hline 10/05/1988 & 1.7 & MERLIN & Spencer et al. 1993 [26] \\
\hline 19/05/1988* & 1.7 & MERLIN & Spencer et al. 1993 [26] \\
\hline 10/06/1988 & 1.7 & MERLIN & Spencer et al. 1993 [26] \\
\hline 28/06/1988 & 1.7 & MERLIN & Spencer et al. 1993 [26] \\
\hline 07/12/1991 & 5 & MERLIN & Stirling et al. 2002 [28] \\
\hline 12/12/1991* & 5 & MERLIN & Stirling et al. 2002 [28] \\
\hline 22/12/1991 & 5 & MERLIN & Stirling et al. 2002 [28] \\
\hline 04/01/1992* & 5 & MERLIN & Stirling et al. 2002 [28] \\
\hline 25/09/1993* & 1.7 & MERLIN & Halai 2001 [8] \\
\hline 19/04/1994 & 1.6 & VLBA & Stirling et al. 1997 [27] \\
\hline 21/04/1994 & 1.6 & VLBA & Stirling et al. 1997 [27] \\
\hline 06/05/1995* & 1.6 & VLBA & Paragi et al. 1999 [17] \\
\hline 15/02/1997 & 5 & MERLIN & Stirling et al 2004 [29] \\
\hline 07/03/1998* & 5 & VLBA/MERLIN & Blundell et al. 2001 [1] \\
\hline 26/03/1998 & 15 & VLBA & Paragi et al. 1999 [17] \\
\hline 18/04/1998 & 22 & VLBA & Paragi et al. 1999 [17] \\
\hline 22/05/1998* & 5 & VLBA & Paragi et al. 2002 [19] \\
\hline 06/06/1998* & 1.7 & EVN/MERLIN & Paragi [19], Stirling [29] \\
\hline 16/06/1998* & 5 & EVN & Paragi et al. 2002 [19] \\
\hline $13 / 02 / 2000 *$ & 1.7 & VLBA/EVN/MERLIN & Paragi et al. 2002 [19] \\
\hline $20 / 02 / 2000^{*}$ & 1.7 & VLBA/EVN & Paragi et al. 2002 [19] \\
\hline 27/03/2000 & 5 & MERLIN & Stirling et al. 2004 [29] \\
\hline $27 / 05 / 2000^{*}$ & 1.7 & VLBA/EVN & Paragi et al. 2002 [19] \\
\hline $14 / 10 / 2001$ & 5 & MERLIN & Stirling et al. 2004 [29] \\
\hline $10 / 07 / 2003$ & 4.9 & VLA & Blundell \& Bowler 2004 [2] \\
\hline 06/08/2003* & 1.5 & VLBA & Mioduszewski et al. in prep \\
\hline
\end{tabular}

\section{The Properties of the Equatorial Emission}

Examination of the images shows that the position angle of the equatorial emission changes from epoch to epoch, for example the emission in figure 2 is in a position angle (PA) of $0 \pm 2^{\circ}$, whereas the emission in the images from the Mioduszewski VLBA [21] movie is in PA $20 \pm 10^{\circ}$. Figure 3 shows a plot of the PA vs modified Julian date (JD-2400000.5). There is no clear periodic pattern, though this not surprising given that the 52 cycles of the 162.5 day precession period in the data are seriously under-sampled. Figure 4 shows a histogram of the positions angles, it is notable that the mean position angle $\left(10.4 \pm 3.0^{\circ}\right)$ is perpendicular to the mean jet axis (PA $100^{\circ}$ ) and with a similar range (-20 to $35^{\circ}$ compared with 80 to $\left.120^{\circ}\right)$. This strongly suggests that the equatorial emission is related in some way to the precessing jets. However figure 5 shows that the observed position angle does not appear to correlate with the position angle of the inner jets at the observation date (using the Margon (1984) [14] ephemeris. 


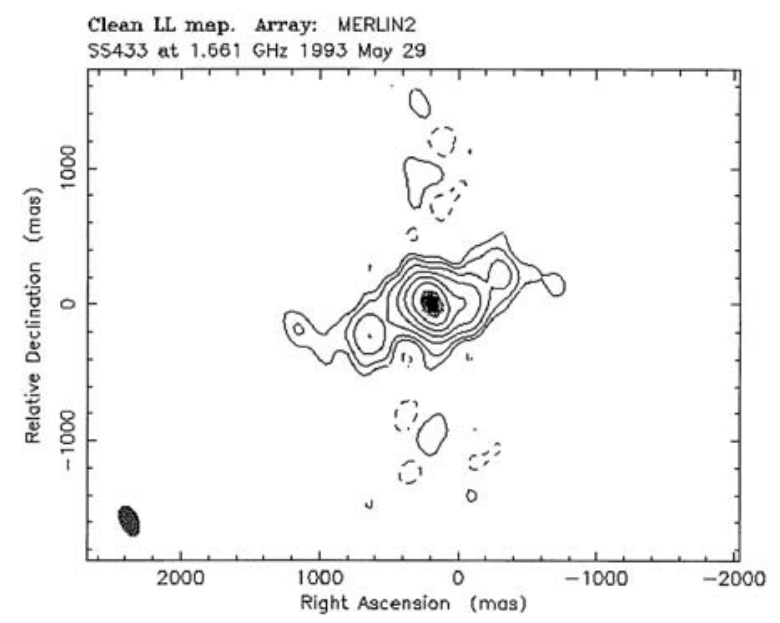

Figure 1 MERLIN image of SS433 at $1.66 \mathrm{GHz}$ taken on 25 October 1993. Note the slight extension to the South of the core. The discrete knots $\sim 1$ arc sec to the $\mathrm{N}$ and $\mathrm{S}$ of the core are likely to be side-lobes and an artefact (from [8]).
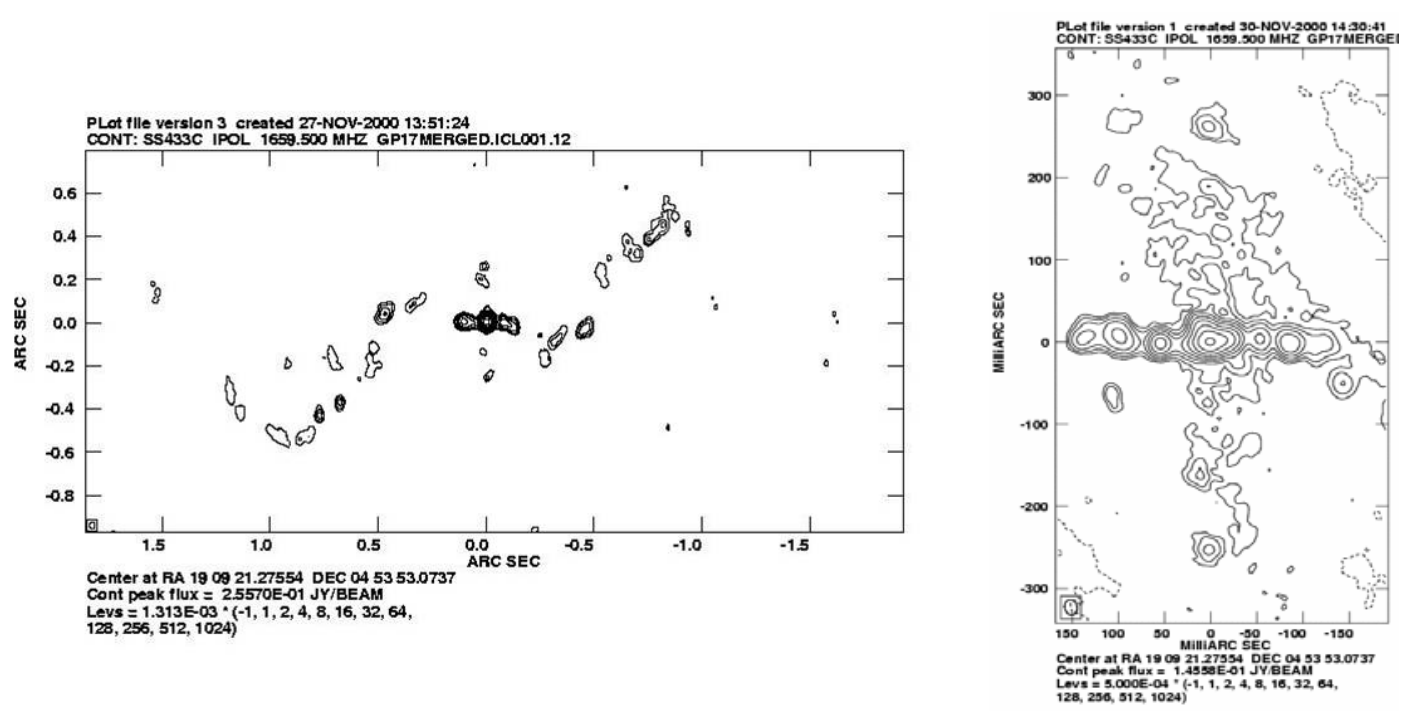

Figure 2 Combined MERLIN and EVN images of SS433 at $1.66 \mathrm{GHz}$, using data taken on 6 June 1998. The map on the left shows the large scale structure, a higher resolution map of the central region is shown on the right. These maps were produced by A. Stirling (priv. comm.) from data also imaged by Paragi et al. 2002 [19] and Stirling et al. 2004 [29]. 


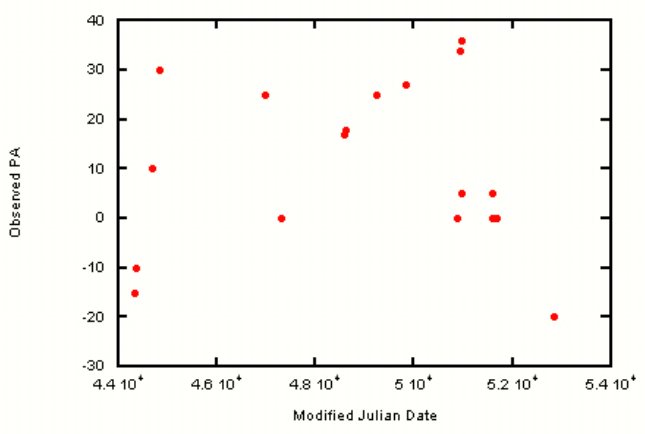

Figure 3 Plot of the position angle of the equatorial emission $v s$ date.

Figure 4 Histogram of the observed position angles of the equatorial emission.

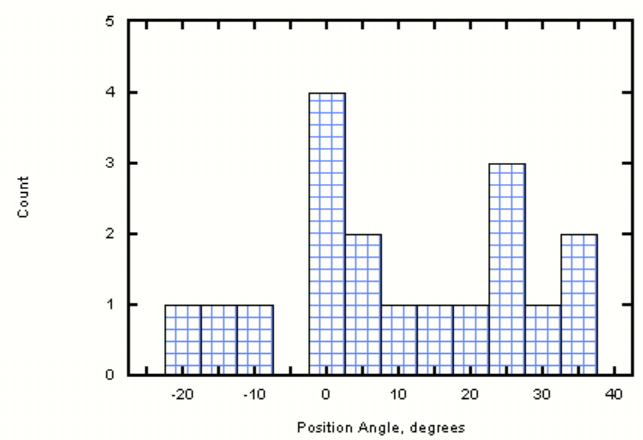

There is also no correlation if the equatorial emission had been ejected at a previous date to the observations: time delays for each observation were calculated from angular size estimates taken from the published images and assuming velocities of 5000 and $10000 \mathrm{~km} \mathrm{sec}^{-1}$. Corresponding inner jet position angles were found from the ephemeris using the corrected date, but comparison with the observed PA also has a random scatter.

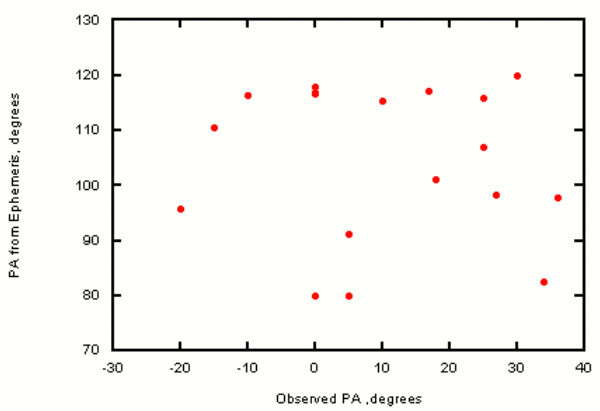

Figure 5 Plot of the observed position angle vs the expected position angle of the inner jets derived from the Margon 1984 ephemeris.

\section{Discussion and Conclusions}

On the precessing jet model [14] the inner disk around the compact star precesses due to tidal torques, and so we would not expect to see precession of an anisotropic wind from the companion star. However, the fact that the position angle does change lends support to the presence of a disk wind [3] where the disk and wind also precess. We would expect to see a delay between the jet ejection angle (and by inference the inner disk PA) and the outer disk PA and disk wind direction. The lack of a clear correlation with the jet direction also suggests that if a wind is indeed the source of the equatorial emission then the wind velocity cannot be constant. 
Examination of the images shows a few cases where we can perhaps relate the position angle of the jet to the observed equatorial emission PA. In figure 2, the equatorial emission is perpendicular to the jet at a position $\sim 0.5$ arc sec from the core. Other cases show that the PAs are perpendicular to each other at distances up to 2 arc sec. These angular separations correspond to delays of 50 to 200 days (given the $0.26 \mathrm{c}$ jet velocity and a distance of $4.8 \mathrm{kpc}$ ) between the ejection of an equatorial wind and hence corresponding velocities of 16000 and $4000 \mathrm{~km} \mathrm{sec}^{-1}$. With such high wind velocities it is not surprising that high brightness nonthermal knots occur, formed by particle acceleration in strong shocks.

An alternative explanation of the equatorial emission comes from hydrodynamic studies of bipolar nebulae [12]. The colliding backflow from ejected clumps in high velocity jets can cause enhanced densities in the equatorial plane (essentially where the two bow shocks from each jet in a two-sided jet structure meet). Simulations have shown that the density enhancement can be high in a circumstellar atmosphere where the density (perhaps in a wind) falls off as the inverse square of the radius (Smponias, in prep.).

Archive data have shown that the anomalous emission aligned roughly along the equator of SS433 was detected in the past. It does not always seem to be present; this could be due to mapping fidelity problems, though the MERLIN observations of Stirling et al. 1992 showed that the emission, though clearly present on 12 December 1991, was not present 10 days later. Also the VLBA movie by Mioduszewski shows variable intensity for the equatorial emission. This emission is undoubtedly a feature of SS433, to add to the list of its bizarre properties.

\section{Acknowledgements}

The author would like to thank Alastair Stirling for valuable discussions, and Gansham Halai who found most of the references for his thesis work.

\section{References}

[1] Blundell, K. M., Mioduszewski, A.J., Muxlow, T.W.B., Podsiadlowski, P., \& Rupen, M.P. 2001, ApJ, 562, L79

[2] Blundell, K. M. \& Bowler, M. G., 2004 ApJ, 616, L159

[3] Fabrika, S.N. 1993, MNRAS, 261, 241

[4] Fejes, I., 1986, A\&A, 168, 69

[5] Fejes, I.; Schilizzi, R. T.; Vermeulen, R. C., 1988, A\&A 189, 124

[6] Gilmore, W., Seaquist, E., 1980, AJ, 85, 1486

[7] Gilmore, W.S., Seaquist, E.R., Stocke, J. T., \& Crane, P.C. 1981, AJ, 86, 864

[8] Halai, G., MSc thesis, Jodrell Bank Observatory, Physics and Astronomy, The University of Manchester, 2001

[9] Heavens, A, F., Ballard, K. R. Kirk, J. G., 1990, MNRAS, 244, 474 
[10] Hjellming, R. M., Johnston, J. K. 1981, Nature, 290, 100

[11] Hjellming, R. M., Johnston, J. K. 1981b, ApJ, 246, L141

[12] Lloyd, H. M., Bode, M.F., O'Brien, T. J., \& Kahn, F.D. 1993, MNRAS, 265, 457

[13] Margon, B., Stone, R. P. S., Klemola, A., Ford, H.C., Katz, J.I., Kwitter, K.B., \& Ulrich, R. K. 1979, ApJ, 230, L41

[14] Margon, B. 1984, ARAA, 22, 507

[15] Niell, A. R., Lockhart, T. G., Preston, R. A., Backer, D. C., in Extragalactic Radio Sources, eds. D. S. Heeschen, C. M., Wade, IAU Symp. 97, 207

[16] Paragi, Z., Vermeulen, R.C., Fejes, I., Schilizzi, R.T., Spencer, R. E., \& Stirling, A.M. 1998, NewAR, 42, 641

[17] Paragi, Z., Vermeulen, R. C., Fejes, I., Schilizzi, R. T., Spencer, R .E., \& Stirling, A. M., 1999, A\&A, 348, 910

[18] Paragi, Z., Fejes, I., Vermeulen, R. C., Schilizzi, R. T., Spencer, R. E., \& Stirling, A.M., 2001, APSS, 276, 131

[19] Paragi, Z., Fejes, I., Vermeulen, R.C., Schilizzi, R. T., Spencer, R. E., \& Stirling, A. M., 2002, Proc. 6th EVN Symp., 263

[20] Romney, J. D., Schilizzi, R. T., Fejes, I., \& Spencer, R. E. 1987, ApJ, 321, 822

[21] Rupen, M., Mioduszewski, A., Taylor,G., Walker, C., NRAO press releases, January 2004

[22] Schilizzi, R. T., Miley, G. K., Romney, J. D. \& Spencer, R. E., 1982, Nature 290, 318

[23] Spencer, R. E. 1979, Nature, 282, 483

[24] Spencer, R.E. 1984, MNRAS, 209, 869

[25] Spencer, R. E., Waggett, P. 1984, in VLBI and Compact Radio Sources, eds. R. Fanti , G. Setti, IAU Symp. 110, 289

[26] Spencer, R.E., Vermeulen, R.C., I\& Schilizzi, R.T. 1993, ASSL Vol. 186: Stellar Jets and Bipolar Outflows, 203

[27] Stirling, A. M., Spencer, R. E., Watson, S. K., 1997, Vistas in Ast. 41, 197

[28] Stirling, A.M., Jowett, F. H., Spencer, R. E., Paragi, Z., Ogley, R. N., \& Cawthorne, T. V., 2002, MNRAS, 337, 657

[29] Stirling, A. M., Spencer, R. E., Cawthorne, T. V. Paragi, Z., 2004, MNRAS 354, 1239

[30] Vermeulen, R. C., Icke, V., Schilizzi, R. T., Fejes, I., \& Spencer, R. E. 1987, Nature, 328, 309

[31] Vermeulen, R., The jets of SS433, in Astrophysical Jets, Burgarella, D., Livio, M., O'Dea, C., (eds.), Space Telescope Science Institute Symposium Series, Cambridge University Press, 1993. 